\title{
Vesicle Origami: Cuboid Phospholipid Vesicles Formed by Template-Free Self-Assembly
}

\author{
Frederik Neuhaus, ${ }^{[a, b, c]}$ Dennis Mueller, ${ }^{[a, c]}$ Radu Tanasescu, ${ }^{[a]}$ Sandor Balog, ${ }^{[\mathrm{d}]}$ Takashi Ishikawa, ${ }^{[\mathrm{e}]}$ \\ Gerald Brezesinski, ${ }^{[f]}$ Andreas Zumbuehl ${ }^{*[a, b]}$
}

\begin{abstract}
Phospholipid liposomes are archetypical self-assembled structures. In order to minimize the surface tension, the vesicles typically are spherical. Deciphering the bilayer code, the basic physical interactions between phospholipids, would allow to utilize these molecules as building blocks for novel, non-spherical structures. Here, we present a 1,2-diamidophospholipid that selfassembles into a cuboid structure. Due to intermolecular hydrogen bonding, the bilayer membranes form an exceptionally tight subgel packing leading to a maximization of flat structural elements and a minimization of any edges. These conditions are optimized in the geometrical structure of a cube. Surprisingly, the lateral surface pressure in the membrane is only one third of the value typically assumed for a bilayer membrane, questioning a long-standing rule of thumbs.
\end{abstract}

Phospholipids form monolayers at the air water interface ${ }^{[1]}$ and selfaggregate into $3 \mathrm{D}$ vesicles in aqueous media ${ }^{[2]}$. The ordering properties of a phospholipid in $2 \mathrm{D}$ layers can act as a first indication of how the compound will behave in a bulk system. ${ }^{[1 \mathrm{c}, 3]}$ Until today only few lipid self-assembly systems are known that depart from the standard spherical vesicle shape ${ }^{[4]}$ and even less such systems are known that are formed from true phospholipids. ${ }^{[5]}$ Very recently, an amphiphilic membrane was spanned over a DNA-origami templated cube. It was mentioned that reaching such membrane structures in a non-templated fashion will be challenging ${ }^{[6]}$ Here, we show how the biophysical properties of a 1,2-diamidophospholipid in a monolayer are reflected in the bilayer by the formation of the first non-templated self-assembled phospholipid cuboid. The synthetic phospholipid

[a] F. Neuhaus, Dr. D. Mueller, R. Tanasescu, Prof. Dr. Andreas Zumbuehl

Department of Chemistry

University of Fribourg

Chemin du Musée 9

1700 Fribourg, Switzerland

E-mail: andreas.zumbuehl@unifr.ch

[b] National Centres of Competence in Research in Chemical Biology

(Geneva) and Bio-inspired Materials (Fribourg)

[c] These authors contributed equally to this work

[d] Dr. S. Balog

Adolphe Merkle Institute

University of Fribourg

Chemin du Verdiers 4

1700 Fribourg, Switzerland

[e] Dr. T. Ishikawa

Paul Scherrer Institute (PSI)

OFLB/010

5232 Villigen PSI, Switzerland

[f] Prof. Dr. G. Brezesinski

Max Planck Institute of Colloids and Interfaces

Research Campus Potsdam-Golm

14476 Potsdam, Germany

Supporting information for this article is given via a link at the end of the document.
Pad-Pad-PC (1) leads to perfect monolayer packing with amideamide hydrogen bonding optimizing the attractive inter-tail hydrophobic forces. Organizing two such stiff membranes into a 3D bilayer vesicle calls for the maximization of flat areas and the minimization of line defects; a cube is formed. The tightness of the vesicles and their mechano-responsiveness are interesting biophysical properties for drug encapsulation and targeted drug delivery. ${ }^{[5]}$ Cracking the bilayer self-assembly code will open a new chapter in phospholipid research where we will be able to guide selfassembly and use phospholipids as a versatile building material akin to how nature is using the phospholipids to construct cells and living systems.

Recently, we have reported that the 1,3-diamidophospholipid Pad-PC-Pad (2) forms interdigitated stiff bilayers and therefore facetted vesicles. ${ }^{[5,7]}$ This caused us to probe the forces at play in membranes of a diamidophospholipid with an 1,2-substitution pattern, Pad-Pad-PC (1) as seen in Figure 2. Changing the substitution pattern of the phospholipid from 1,3 to a 1,2 should not allow for membrane interdigitation but should keep the continuous amide-amide hydrogen bond pattern in the membrane backbone. The molecule Pad-Pad-PC (1) has been synthesized before as an anti-HIV drug ${ }^{[8]}$ and we have studied the vesicle's limited mechanoresponsiveness. ${ }^{[5]}$ Here, we have resynthesized Pad-Pad-PC (1) at very high chemical purity and for the first time analyzed its biophysical properties.

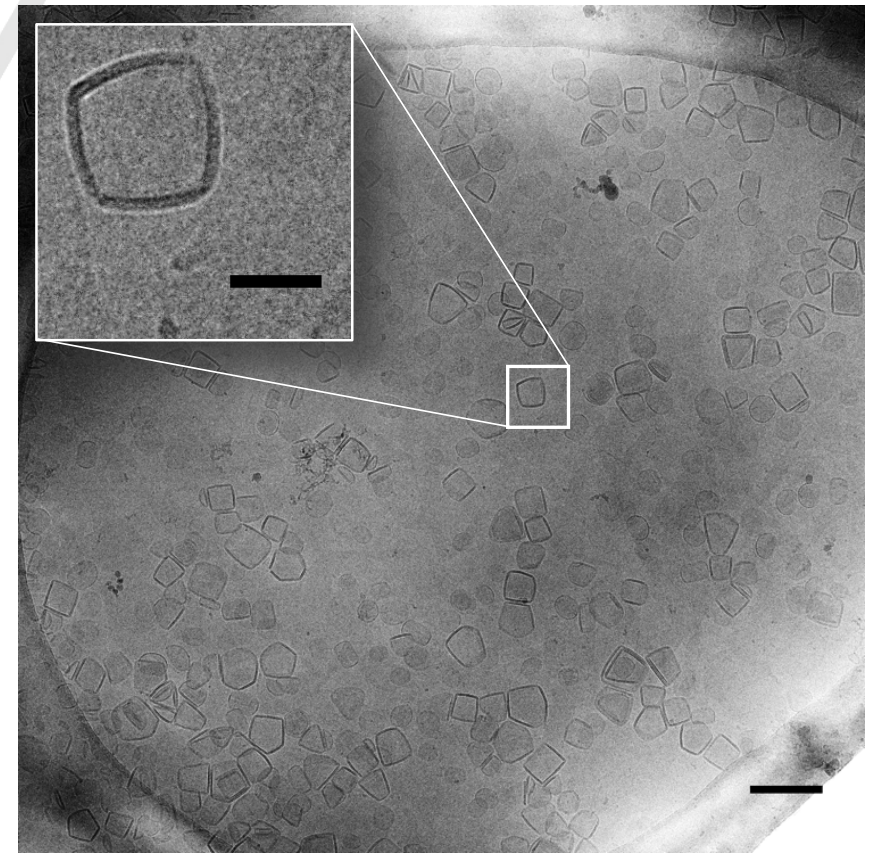

Figure 1. Cryo-transmission-electron-microscopy image of Pad-Pad-PC (1) vesicles (scale bar $=200 \mathrm{~nm}$ ). The inset (scale bar $=50 \mathrm{~nm}$ ) clearly shows cuboid vesicles with a continuous outer membrane leaflet and defects in the inner leaflets at the edges. 

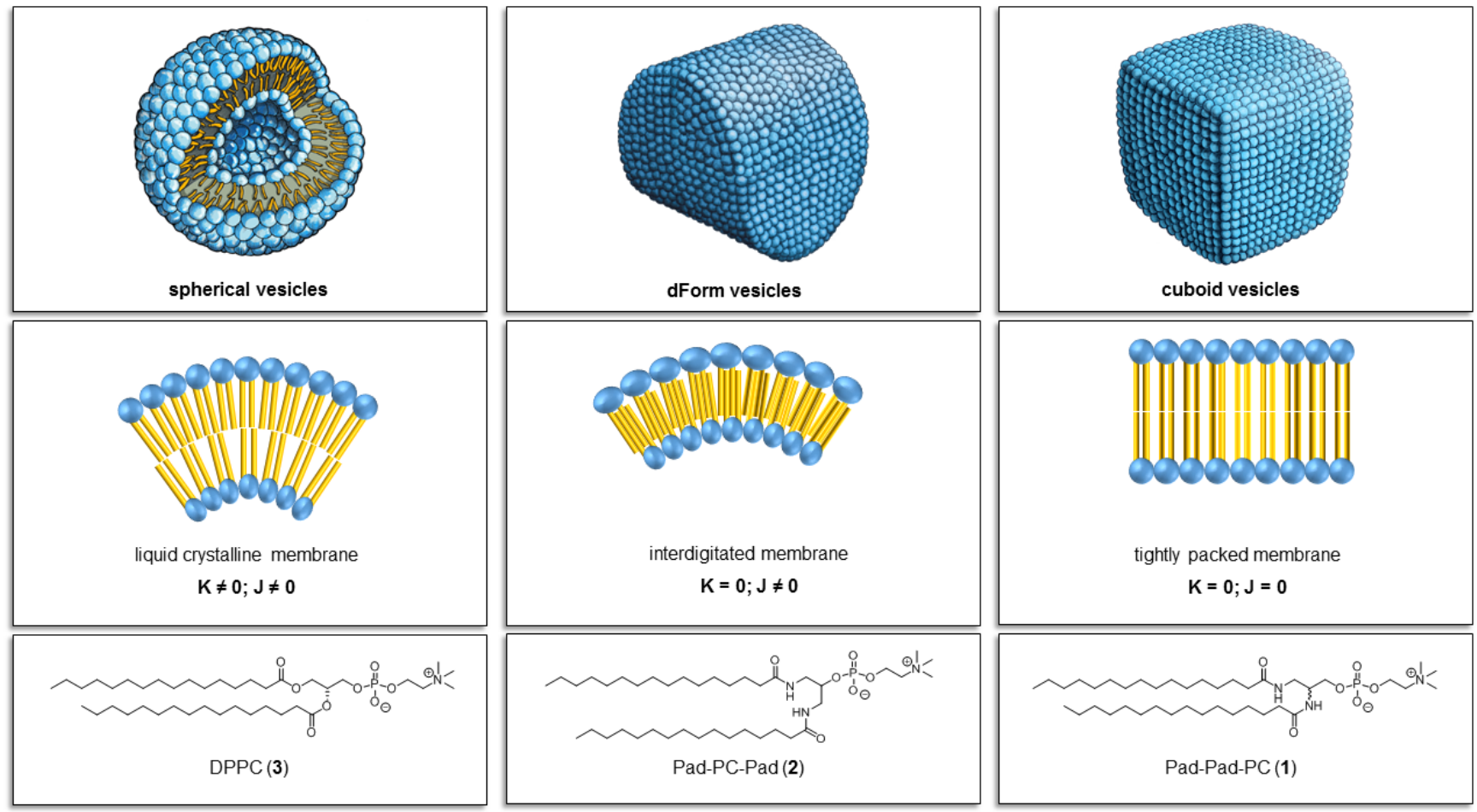

Figure 2. Dependence of the geometrical shape of the liposomes on the intrinsic Gaussian curvature K and the extrinsic total curvature J. All systems were initially formulated above the main phase transition temperature $\left(T_{m}\right)$ of the phospholipids, where the liquid-crystalline $L_{\alpha}$ phase places no constraint on the vesicle geometry and all vesicles are spherical. Upon cooling below $T_{m}$, the intrinsic and extrinsic curvatures of the bilayers force the spheres into alternative shapes. The vesicles are formed from one single type of phospholipid only. The chemical structures are depicted at the bottom of the figure.

pattern in the membrane backbone. The molecule Pad-Pad-PC (1) has been synthesized before as an anti-HIV drug ${ }^{[8]}$ and we have studied the vesicle's limited mechano-responsiveness. ${ }^{[5]}$ Here, we have resynthesized Pad-Pad-PC (1) at very high chemical purity and for the first time analyzed its biophysical properties.

Large unilamellar vesicles (LUVET 100 ) were obtained by the classical thin film hydration and extrusion method in saline. ${ }^{[9]}$ After the vesicle were formulated above the phase transition temperature, the liposomes were cooled down to room temperature, below the phase transition temperature. The cryo transmission electron micrograph (cryo-TEM, see Figure 1) of Pad-Pad-PC (1) vesicles surprisingly shows highly facetted aggregates of unprecedented cuboid shape, more clearly visible in the cryo-TEM tomogram (see supporting information). The vesicle cubes have a face length of $60 \mathrm{~nm}$. Using the area per tail of $0.191 \mathrm{~nm}^{2}$ (see supporting information) means that $11 \mathrm{x}$ $10^{4}$ lipids self-assemble into one vesicle with a mass of approximately $100 \mathrm{ag}$. We cannot exclude that smallest sample impurities are causing distortion from the cuboid geometry. Overall, we measure $45 \%$ cuboid objects, $33 \%$ highly faceted objects and $22 \%$ nonfaceted objects. The orientation of the cuboids in space seems mostly random (see supporting information). Crowding of the vesicles might lead to side-by-side stacking with more structures standing flat on the grid. This orientation is expected to being overemphasized in Figure 1 because of the higher sampling along the vertical cuboid faces compared to the horizontal faces.

The presence of a tight hydrogen bonding network between the Pad-Pad-PC (1) amide bonds is clearly visible in the wide-angle X-ray scattering (WAXS) at $1.33 \AA^{-1}$, which represents the same repeating distance of $4.75 \AA$ that is seen between hydrogen bonded strands of a peptide $\beta$-sheet (see supporting information). ${ }^{[10]}$ This network leads to a complex melting behavior in differential scanning calorimetry (see supporting information) with a main $T_{m}$ at around $46{ }^{\circ} \mathrm{C}$, which is significantly higher compared to the $\mathrm{T}_{\mathrm{m}}$ of $37^{\circ} \mathrm{C}$ for Pad-PC-Pad (2) and $41.5{ }^{\circ} \mathrm{C}$ for DPPC (3), and additionally hints at a lower hydration of the Pad-Pad-PC head group compared to the other molecules. Measuring the membrane thickness from the cryoTEM image (see supporting information) of three different vesicles at a total of 72 cross-sections we do get an average value of $6.76 \pm 0.17 \mathrm{~nm}$. This agrees well with the d-values for the lamellar phases $\left(6.85 \mathrm{~nm}\right.$ at $18{ }^{\circ} \mathrm{C}$ and $6.99 \mathrm{~nm}$ at $\left.50{ }^{\circ} \mathrm{C}\right)$ observed in small-angle X-ray scattering (SAXS) measurements (see supporting information). The calculated bilayer membrane thickness for the lipid with fully extended all-antiperiplanar fatty acid chains and an extended head group, is $6.80 \mathrm{~nm},{ }^{[7 b]}$ which calls for a non-interdigitated membrane with lipids oriented parallel to the membrane normal. Furthermore, the WAXS traces suggest the tightest bilayer packing possible, a herringbone packing with values of $0.19 \mathrm{~nm}^{2}$ which are in line with a subgel phase, which for natural phospholipids (3) is only visible after cooling for several days ${ }^{[11]}$. Upon lipid melting, the hydrogen bonding network will be destroyed increasing the intermolecular distance. This leads to a much larger cross-sectional area of the lipid chains $\left(0.26 \mathrm{~nm}^{2}\right)$ which should lead to a thinning of the bilayer but is overcompensated by the now better head group hydration and the possible increase of the interbilayer water layer.

DPPC (3) forms only mildly facetted vesicles in the gel phase. The herringbone packing of the lipids is not found for DPPC and is most likely induced by the additional hydrogen bonding network present in Pad-Pad-PC. The additional interaction leads to a minimization of the intermolecular distance and thus to a maximization of the chain-chain London dispersion forces. The head groups can stand up, visible in the two 
electron-dense layers of the cube-bilayers in cryo-TEM and the white halo around them.

The tight phospholipid packing should stiffen the membrane so much that the intrinsic Gaussian curvature becomes zero. ${ }^{[12]}$ We have already seen this when preparing vesicles of Pad-PC-Pad (2) where the restricted curvature led to a dForm vesicle, a developable surface made from two pieces of ellipsoidal bilayers. In a dForm vesicle, the total curvature is nonzero, allowing for the rounded geometrical shape. ${ }^{[\mathrm{b}]}$ Compared to Pad-PC-Pad (2), a vesicle membrane made from Pad-Pad-PC (1), is even stiffer. This leads to both a Gaussian and a total curvature of zero. At no point on the membrane, a curvature should be allowed. The geometrical shape of the PadPad-PC (1) vesicles therefore must still be developable and the ratio between flat faces and edges has to be maximized, which leads to a cuboid structure.

The formulation of the Pad-Pad-PC (1) vesicles was performed above the phase transition temperature where the membrane is in a fluid state and the vesicles can adopt a spherical geometry. Cooling the vesicles down below the $T_{m}$ results in a phase change into a subgel phase and the system has to adopt to the new constraints given by the stiff membranes: the cuboid is formed but as a consequence the inner bilayer membrane must be discontinuous at the edges (see Figure 1). The outer membrane is curved over the edges and leads to a closed 3D structure. The cuboid is fairly tight and only about a fifth of the encapsulated 5(6)-carboxyfluorescein was released over the time course of a week. However, the edges of the cuboid are in fact membrane defects that will be attenuated when the vesicles are vigorously shaken. Indeed, a mild mechanoresponse was found and the dye release after one minute of shaking was one fourth of the release recorded for the Pad-PC-Pad (2) vesicles (see supporting information). Being able to release an encapsulated dye molecule upon an external mechanical trigger, e.g. increased wall shear stresses, is a promising concept for targeted drug delivery. ${ }^{[5,13]}$

In order to gain additional insight into the denser-thanusual packing of the fatty acid chains, we have measured surface-pressure/molecular area isotherms at the soft air/water interface (see supporting information). It showed a lift-off from a relatively small molecular area of around $60 \AA^{2}$ and culminated in a very condensed phase that is seldom seen for PC lipids. The different phases were characterized by grazing incidenceangle X-ray diffraction (GIXD). ${ }^{[\mathrm{b}]}$ At $10{ }^{\circ} \mathrm{C}$ and $15{ }^{\circ} \mathrm{C}$ and low lateral pressures of $5 \mathrm{mN} / \mathrm{m}$ and $10 \mathrm{mN} / \mathrm{m}$ a very tight packing in a $L_{2}^{\prime}$ phase with cross-sectional areas of $19.2 \AA^{2}$ per chain was found. This tight membrane packing is destroyed upon further compression and an $L_{2}$ rotator phase is found at higher pressures. The unit cell changes its main distortion direction from NNN (next nearest neighbour) to NN (nearest neighbour) at increasing surface-pressures. At the same time the tilt switches direction from NNN to NN. ${ }^{[14]}$ While the tilt direction changes, the tilt angle, relative to the monolayer normal, stays nearly the same as shown in Figure 3 . The system is characterized by second-order phase changes until finally the LS phase with untilted chains is reached. The smallest chain diameters are found for low surface pressures.

At higher temperatures, the $L_{2}^{\prime}$ phase disappears and is replaced with a region lacking $X$-ray diffraction signals because the thermal energy is high enough to induce defects in the hydrogen-bonding network, resulting in a reduction of the correlation length. Upon monolayer compression, the defects are reduced and the membrane passes an $\mathrm{Ov}$ and $\mathrm{L}_{2}$ phase before ending in the LS phase.

At surface pressures above $30 \mathrm{mN} / \mathrm{m}$ a LS phase is observed. In this rotator phase the fatty acid chains order in a hexagonal unit cell parallel to the membrane normal. ${ }^{[14]}$ The cross-sectional area of each Pad-Pad-PC (1) chain in this hexatic phase amounts to $20.1 \AA^{2}$. Untilted chains have never been observed in natural PC phospholipids. ${ }^{[15]}$ Even the very tightly packed dihexadecyl phosphatidylcholine supports a higher in-plane area $\left(21.8 \AA^{2}\right)$ and a tilt angle of $21^{\circ} .^{[15 a]}$ The diester phospholipid DPPC (3) has an in-plane area per chain of $22.4 \AA^{2}$ and a tilt angle of $25^{\circ} .{ }^{[15 a]}$ The molecular area of a PC head group of $\sim 45 \AA^{2}$ underlines our observation that the head group of Pad-Pad-PC (1) orients parallel to the membrane normal due to strong head-head interactions allowing a molecular in-plane are of $40.2 \AA^{2}$ with untilted chains. ${ }^{[16]}$

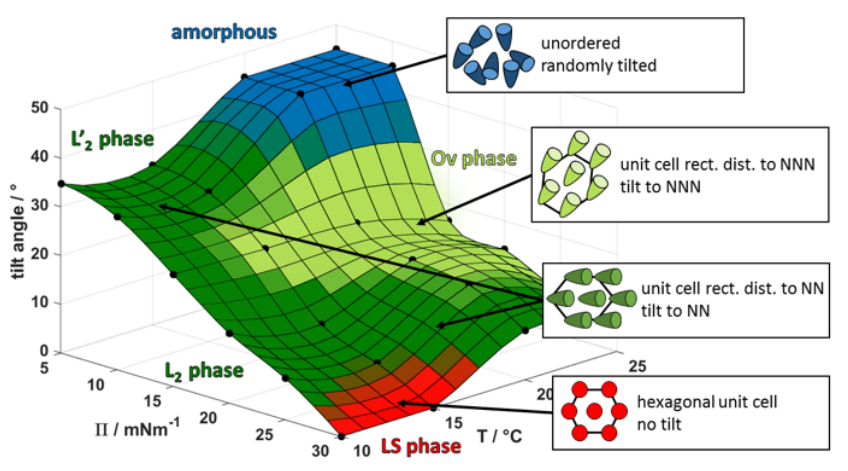

Figure 3. Surface plot of the chain tilt angle of a Pad-Pad-PC (1) monolayer dependent on the temperature and surface pressure. Tilt angles were calculated from GIXD measurements on a Langmuir-Pockels trough using high-brilliance synchrotron irradiation. The surface has an enlarged dataset of a second-generation cubic interpolation. Original fixed data-points are marked as bold black-dots.

Upon compression of the monolayer, the hydrogen bonding network found at low surface pressures $(5-10 \mathrm{mN} / \mathrm{m})$, responsible for the tight packing (19.2 $\AA^{2}$ per chain) will be broken, and the chains organize into a rotator phase at higher cross-sectional area per chain $\left(20.1 \AA^{2}\right)$. In the bulk system, however, a subgel herringbone packing is found $\left(19.1 \AA^{2}\right.$ per chain). This would place the surface pressure of a Pad-Pad-PC (1) bilayer at $5-10 \mathrm{mN} / \mathrm{m}$, which is significantly different from the value of $30 \mathrm{mN} / \mathrm{m}$ generally assumed for the lateral pressure of a bilayer membrane and would question the generality of this rule of thumb. ${ }^{[17]}$

In conclusion, we have demonstrated the formation of very tightly packed membranes containing the single synthetic phospholipid Pad-Pad-PC (1). Such a membrane cannot be bent easily and both the intrinsic Gaussian curvature $K$ and the extrinsic total curvature $\mathrm{J}$ are expected to be zero leading to flat bilayer sheets. ${ }^{[12]}$ These constraints are not present above the $T_{m}$ where the phospholipids can easily be formulated into a 3D system. Upon cooling, the membrane phospholipids again pack into a herringbone structure and the vesicles relax into a shape that maximizes flat faces and minimizes defect lines; a cuboid. Surprisingly, the lateral pressure inside a cubic bilayer must be very low $\left(5-10 \mathrm{mN} / \mathrm{m}\right.$ at $\left.18{ }^{\circ} \mathrm{C}\right)$, contradicting the current belief 
that bilayer membranes always have a lateral pressure of $30 \mathrm{mN} / \mathrm{m}$.

\section{Acknowledgements}

The authors thank Dr. Uta Ruett and Dr. Florian Bertram (Deutsches Elektronen Synchrotron (DESY) Hamburg) for their support and guidance performing the GIXD measurements at PETRA III, P08. This work has been supported by the University of Fribourg and the Swiss National Science Foundation through a stipend professorship to A.Z. and through the National Centres of Competence in Research (NCCR) in Chemical Biology and Bio-inspired Materials.

Keywords: phospholipids • self-assembly $\cdot$ vesicle origami • cuboid vesicles $\cdot$ membrane tension

[1] a) A. Pockels, Nature 1891, 43, 437-439; b) I. Langmuir, J. Am. Chem. Soc. 1916, 38, 2221-2295; c) J. J. Giner-Casares, G. Brezesinski, H. Möhwald, Curr. Opin. Colloid Interface Sci. 2014, 19, 176-182.

[2] a) A. D. Bangham, R. W. Horne, J. Mol. Biol. 1964, 8, 660-668; b) D. D. Lasic, Biochem. J. 1988, 256, 1-11.

[3] a) B. Moghaddam, M. H. Ali, J. Wilkhu, D. J. Kirby, A. R. Mohammed, Q. Zheng, Y. Perrie, Int. J. Pharm. 2011, 417, 235-244; b) L. A. Fedotenko, C. Stefaniu, G. Brezesinski, A. Zumbuehl, Langmuir 2013, 29, 94289435.

[4] a) M. Dubois, B. Demé, T. Gulik-Krzywicki, J. C. Dedieu, C. Vautrin, S. Désert, E. Perez, T. Zemb, Nature 2001, 411, 672-675; b) J. Zhang, G. Xu, A. Song, L. Wang, M. Lin, Z. Dong, Z. Yang, Soft Matter 2015, 11, 7143-7150.

[5] M. N. Holme, I. A. Fedotenko, D. Abegg, J. Althaus, L. Babel, F. Favarger, R. Reiter, R. Tanasescu, P.-L. Zaffalon, A. Ziegler, B. Müller, T. Saxer, A. Zumbuehl, Nat. Nanotechnol. 2012, 7, 536-543.

[6] Y. Dong, Y. R. Yang, Y. Zhang, D. Wang, X. Wei, S. Banerjee, Y. Liu, Z. Yang, H. Yan, D. Liu, Angew. Chem. Int. Ed. 2017, 56, 1586-1589.

[7] a) A. Weinberger, R. Tanasescu, C. Stefaniu, I. A. Fedotenko, F. Favarger, T. Ishikawa, G. Brezesinski, C. M. Marques, A. Zumbuehl, Langmuir 2015, 31, 1879-1884; b) R. Tanasescu, M. A. Lanz, D. Mueller, S. Tassler, T. Ishikawa, R. Reiter, G. Brezesinski, A. Zumbuehl, Langmuir 2016, 32, 4896-4903.

[8] a) C. Jia, A. H. Haines, J. Chem. Soc. Perkin Trans 1 1993, 25212523; b) H.-S. Byun, R. Bittman, J. Org. Chem. 1996, 61, 8706-8708.

[9] P. Walde, in Encyclopedia of Nanoscience and Nanotechnology, Vol. 9 (Ed.: H. S. Nalwa), American Scientific Publishers, Valencia, California, 2004, pp. 43-79.

[10] N. R. Chevalier, C. Chevallard, M. Goldmann, G. Brezesinski, P. Guenoun, Cryst. Growth Design 2012, 12, 2299-2305.

[11] a) J. Katsaras, V. A. Raghunathan, E. J. Dufourc, J. Dufourcq, Biochemistry 1995, 34, 4684-4688; b) D. Marsh, Chem. Phys. Lipids 2012, 165, 59-76.

[12] a) W. Helfrich, Z. Naturforsch. C. 1973, 28, 693-703; b) J. Zimmerberg, M. M. Kozlov, Nat. Rev. Mol. Cell Bio. 2006, 7, 9--19.

[13] a) N. Korin, M. J. Gounis, A. K. Wakhloo, D. E. Ingber, Jama Neurology 2015, 72, 119-122; b) J. Wang, J. A. Kaplan, Y. L. Colson, M. W. Grinstaff, Adv. Drug Deliv. Rev. 2017, 108, 68-82.

[14] V. M. Kaganer, H. Möhwald, P. Dutta, Rev. Mod. Phys. 1999, 71, 779819.

[15] a) H. Möhwald, H. Baltes, M. Schwendler, C. A. Helm, G. Brezesinski, H. Haas, Jpn. J. Appl. Phys. 1995, 34, 3906-3913; b) G. Brezesinski, A. Dietrich, B. Struth, C. Bohm, W. G. Bouwman, K. Kjaer, H. Mohwald, Chem. Phys. Lipids 1995, 76, 145-157.

[16] G. Pabst, S. Danner, S. Karmakar, G. Deutsch, V. A. Raghunathan Biophys. J. 2007, 93, 513-525.

[17] a) A. Blume, Biochim. Biophys. Acta 1979, 557, 32-44; b) D. Marsh, Biochim. Biophys. Acta 1996, 1286, 183-223. 
Entry for the Table of Contents (Please choose one layout)

Layout 1:

\section{COMMUNICATION}

Cuboid Vesicles. A 1,2-diamidophospholipid self-assembles into the first non-template soft matter cubes

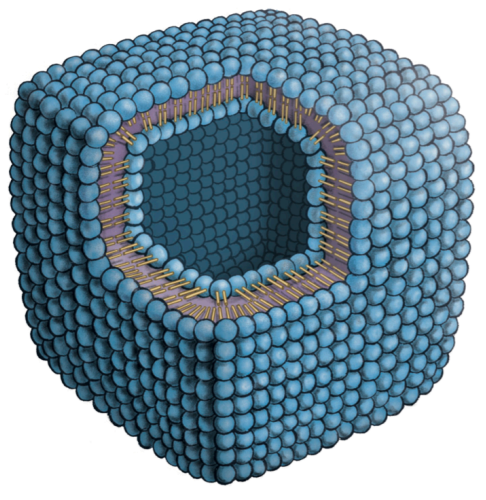

Frederik Neuhaus, Dr. Dennis Mueller, Radu Tanasescu, Dr. Sandor Balog, Dr. Takashi Ishikawa, Prof. Dr. Gerald Brezesinski, Prof. Dr. Andreas Zumbueh/*

Page No. - Page No.

Vesicle Origami: Cuboid Phospholipid Vesicles Formed by Template-Free Self-Assembly 